\title{
High speed visualization of gasoline pump injector (GPI)
}

\author{
Balasubramanian Nallannan ${ }^{1 *}$, Titus Iwaszkiewicz ${ }^{2}$, Jayabalan Sethuraman ${ }^{3}$ \\ 13 Stanadyne India Private Ltd, Thiruvallur 602025, India \\ ${ }^{2}$ Stanadyne LLC, 92 Deerfield Road,Windsor, CT 06095. USA \\ *Corresponding author: nbalasubramanian@stanadyne.com
}

\begin{abstract}
Two-wheeler engines still use carburetor as a fuelling system in many Asian countries, owing to its low cost and less maintenance. The usage of carburetor to handle the upcoming stringent emission norms gets difficult, due to the absence of a closed-loop fuel correction. An electronic fuel injector (EFI), on the other hand, with the help of an electronic control unit (ECU), can correct the fuel quantity and set the air-fuel mixture close to stoichiometric, based on the feedback obtained from the oxygen sensor placed in the exhaust pipe. In this context, an innovative injection system has been developed, that can be applied for such electronic fuel injection in two-wheelers. In this design, the pump and injector are integrated into a single unit, making the system, simple, compact and less expensive. The integrated injector uses a solenoid and spring arrangement, for pressurizing the fuel in a small chamber, and the pressurized fuel is then injected through orifices to produce spray in the intake port. Two-wheeler engines can operate in the order of $10,000 \mathrm{rpm}$ and it poses a big challenge in such injector designs, and therefore the time response of the mechanical and magnetic components of the injector become critical. High-speed back-lit imaging helps in understanding the time response of such injector, by visualizing the spray, while injecting continuously over a period of time. This paper presents the results of high-speed images, obtained from the spray of this new-concept gasoline pump injector (GPI). This exercise, demonstrated that this injector can work at a frequency as high as 83 $\mathrm{Hz}$ and also consistently. The spray pattern was found to be very unique and different from the conventional PFI injection sprays.
\end{abstract}

\section{Keywords}

Port fuel injection, Spray visualization, solenoid pump injector

\section{Introduction}

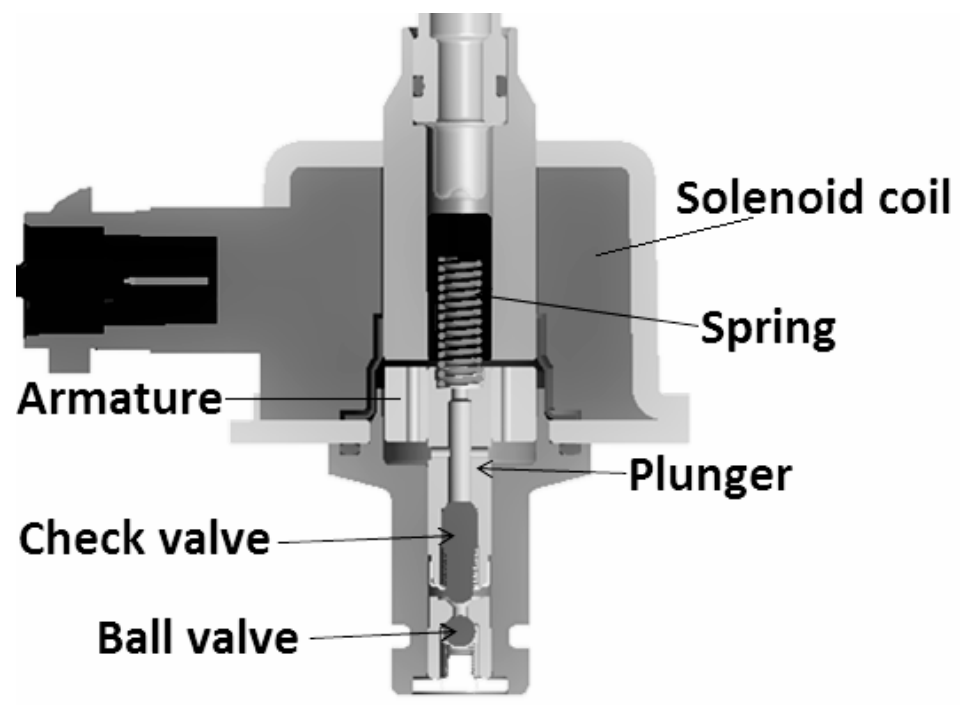

Figure 1. Figure showing the construction of gasoline pump injector (GPI).

The conventional port fuel injector (PFI) receives pressurized fuel, through a pipeline, from an electric rotary pump placed externally. The injector injects the pressurized fuel as and when the electronic control unit (ECU) demands. The pressure of the pump is maintained using an external pressure regulator. The injected fuel quantity is adjusted using pulse width control, to maintain the exhaust lambda values, close to stoichiometric, required for optimum catalytic converter efficiency. This system consumes power continuously as its pump runs continuously and also occupies more space including that of fuel tank's, due to the presence of multiple components discussed. Ways to simplify the injection system have been tried out through several techniques over decades, and one such technique which uses an electromagnetic reciprocating pump to directly displace the fuel is discussed by Heimberg et al.[1] 


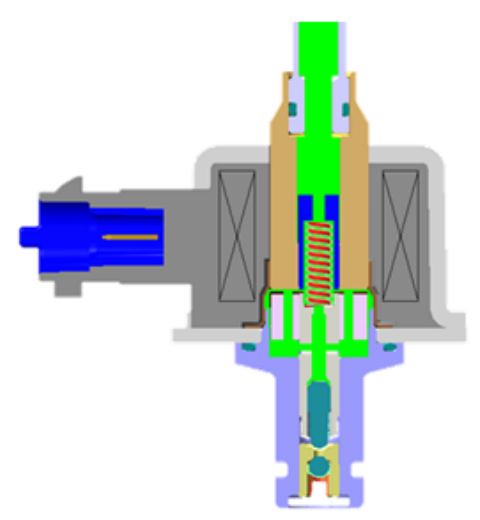

Suction

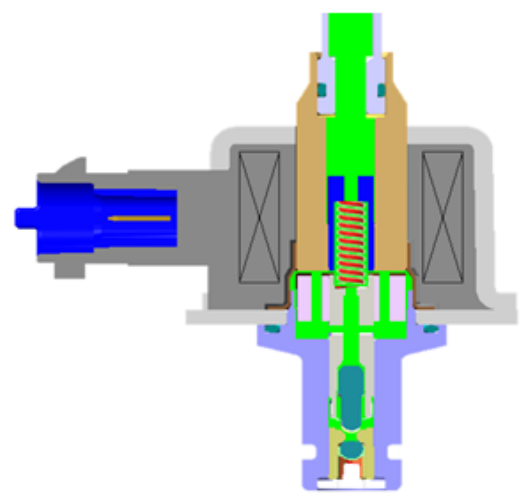

Filling

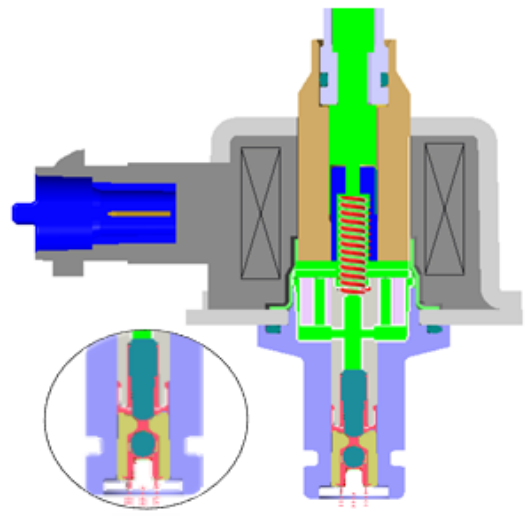

Pumping

Figure 2. Figure showing the working of GPI at various stages.

A similar pump-integrated design has also been invented for after treatment solutions, authored by our colleagues Cavanagh et al.[2] [3], and the work presented in this paper is an extension of that work, to apply the pump-integrated injector on a two-wheeler engine for port fuel injection (PFI). More specifically, this paper studies the time response of the GPI injector, which is very critical as its application on two-wheeler engine, demands faster injector response, owing to shorter cycle times at higher engine rpms.

The construction details of the gasoline pump injector (GPI) are shown in Fig. 1. The GPI is a reciprocating pump and is driven by an electromagnetic solenoid. Upon energizing the solenoid, a magnetic flux is generated around the coil, which pulls the armature up against the spring force. A plunger attached to the armature also moves along with it. A check valve assembly is placed below the plunger also moves along with the plunger. The check valve assembly consists of a check valve loaded by a spring, both placed below the plunger. The check valve moves with a lag, owing to the low pressure produced in the chamber, below the check valve. This low pressure is caused by the increase in the volume produced between the plunger bottom and the ball valve top, when the plunger moves upward, which is shown as 'Suction' in Fig. 2. The effective volume available between the bottom of the check valve and the ball valve top is essentially the 'pumping chamber' of the injector. The low pressure generated in the pumping volume allows the fuel to pass through the clearance between the plunger and the check valve, during the upward motion of the plunger, which is shown as 'Filling' in Fig. 2. When the solenoid is de-energized, the plunger moves down owing to the spring force acting on it. The check valve connected to the plunger gets closed and pressure is generated in the pumping volume, which is shown as 'Pumping' in Fig. 2. Balasubramanian et al. [4] has studied and reported the hydraulic characteristics of this injector in detail.

\section{Methodology}

The spray from the injector was investigated using high speed imaging technique and the test arrangements used are shown in Fig. 3 and Fig. 4. The fuel is fed from a fuel tank, by gravity, to the injector through a filter, as shown in Fig. 3. The injector is placed inside the visualization chamber. The spray zone is back lit using a halogen lamp. A high speed camera of Photron make captures the images from the front side. The injector is controlled by National Instruments' Calview software as shown in Fig. 4, where in the current profile and injection timings are set. The camera is controlled using camera manufacturer's (Photron's) software itself. Necessary pixel-size calibration is done prior to the spray imaging using a syringe-needle arrangement, whose dimensions are known. Upon calibration, the image is seen to occupy 15.9 pixels per $\mathrm{mm}$ of length. The size of the images obtained were of 256 x 472 pixels. The obtained images are post processed using Matlab code, where the images are cropped, background subtracted and finally converted to binary images using Otsu's algorithm. 


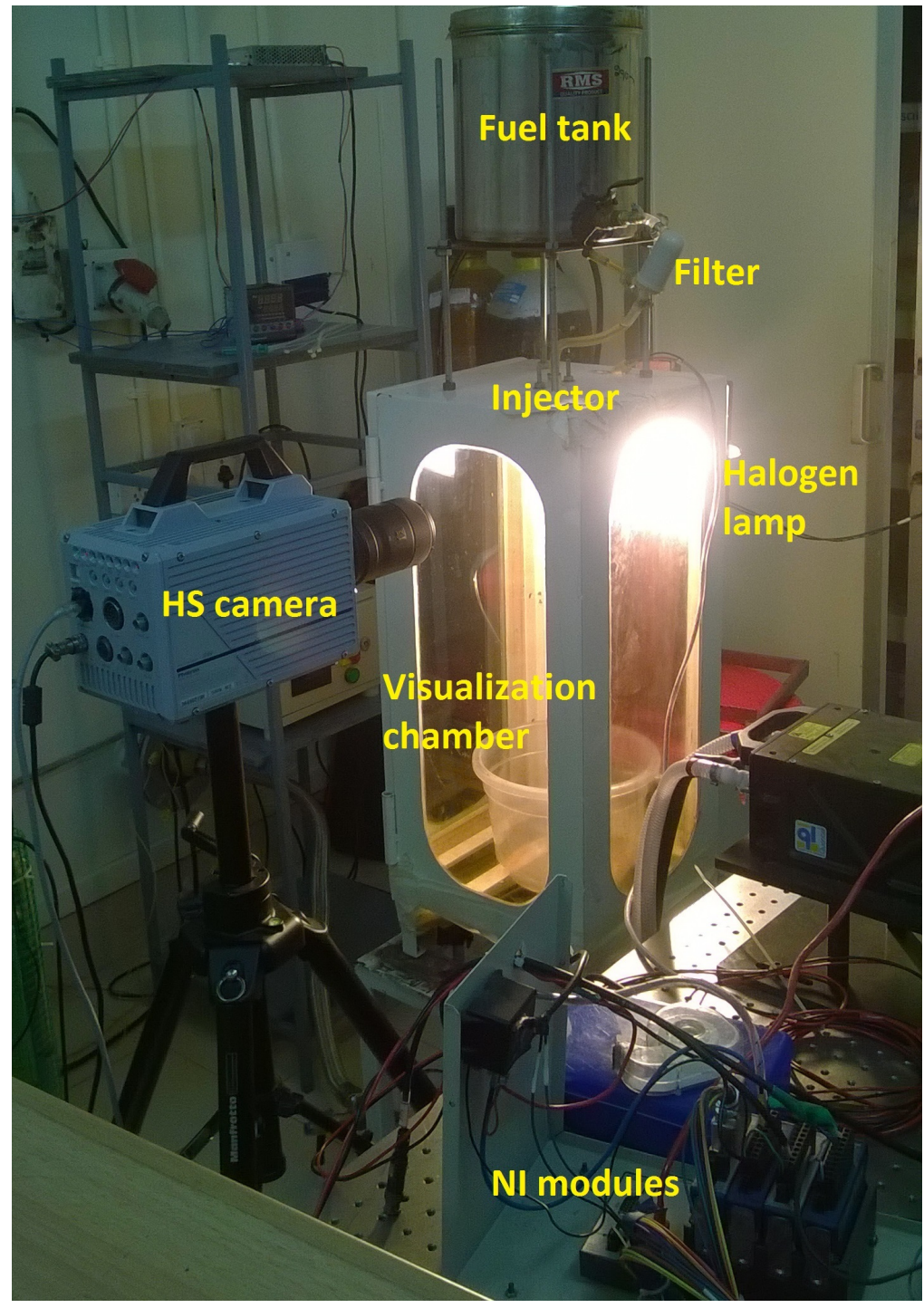

Figure 3. Photograph of the high speed imaging setup

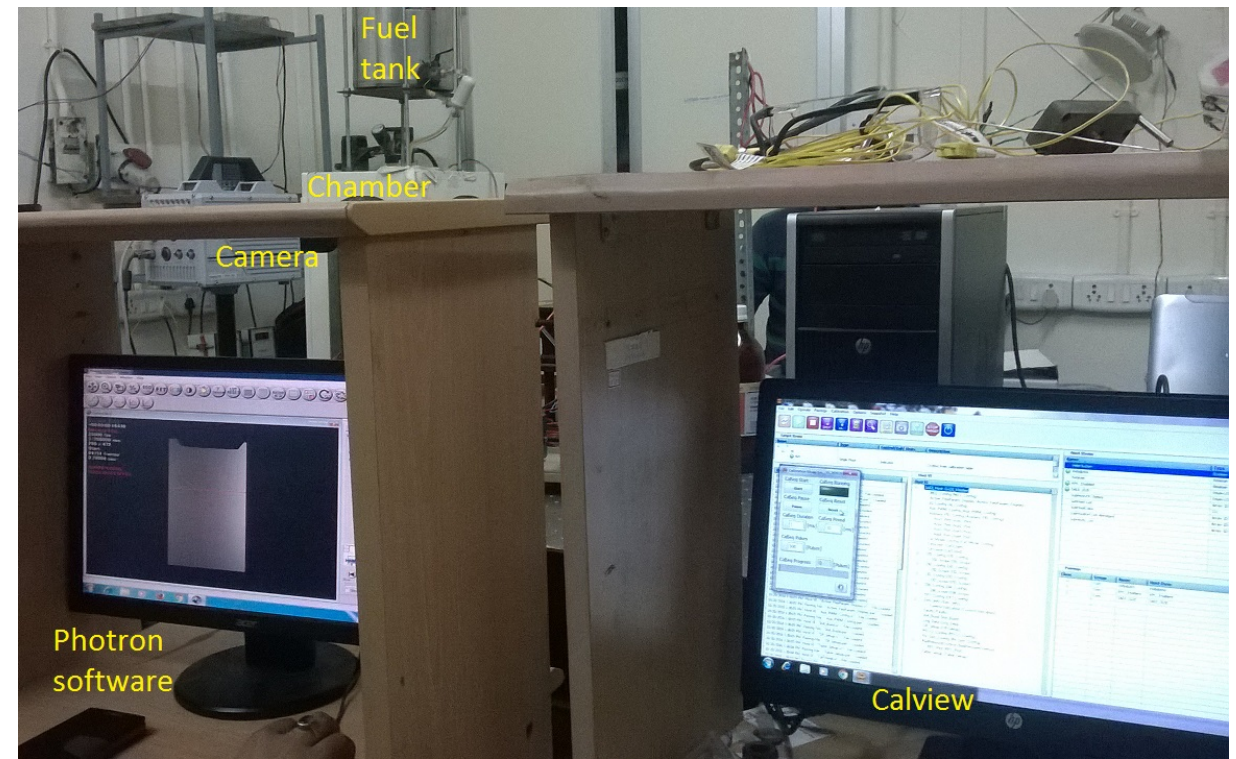

Figure 4. Photograph showing the computer control arrangements for camera and injector 


\section{Results and discussion}

The high speed images were acquired at the rate of $25000 \mathrm{fps}$ (at a time step of $40 \mu \mathrm{s}$ ). The images were continuously taken for two seconds. The injector was triggered at a frequency of $83 \mathrm{~Hz}$ (corresponding to cycles of 10,000 rpm of a 4 stroke engine). All the images obtained during these two seconds were analyzed individually, and confirmed that the injection events were consistent without missing of any cycle. The images captured in consecutive cycles at every $12 \mathrm{~ms}$ time (cycle time)gap are shown in Fig. 5. These images of eight consecutive cycles also correspond to $1.36 \mathrm{~ms}$ after the start of injection(SOI).
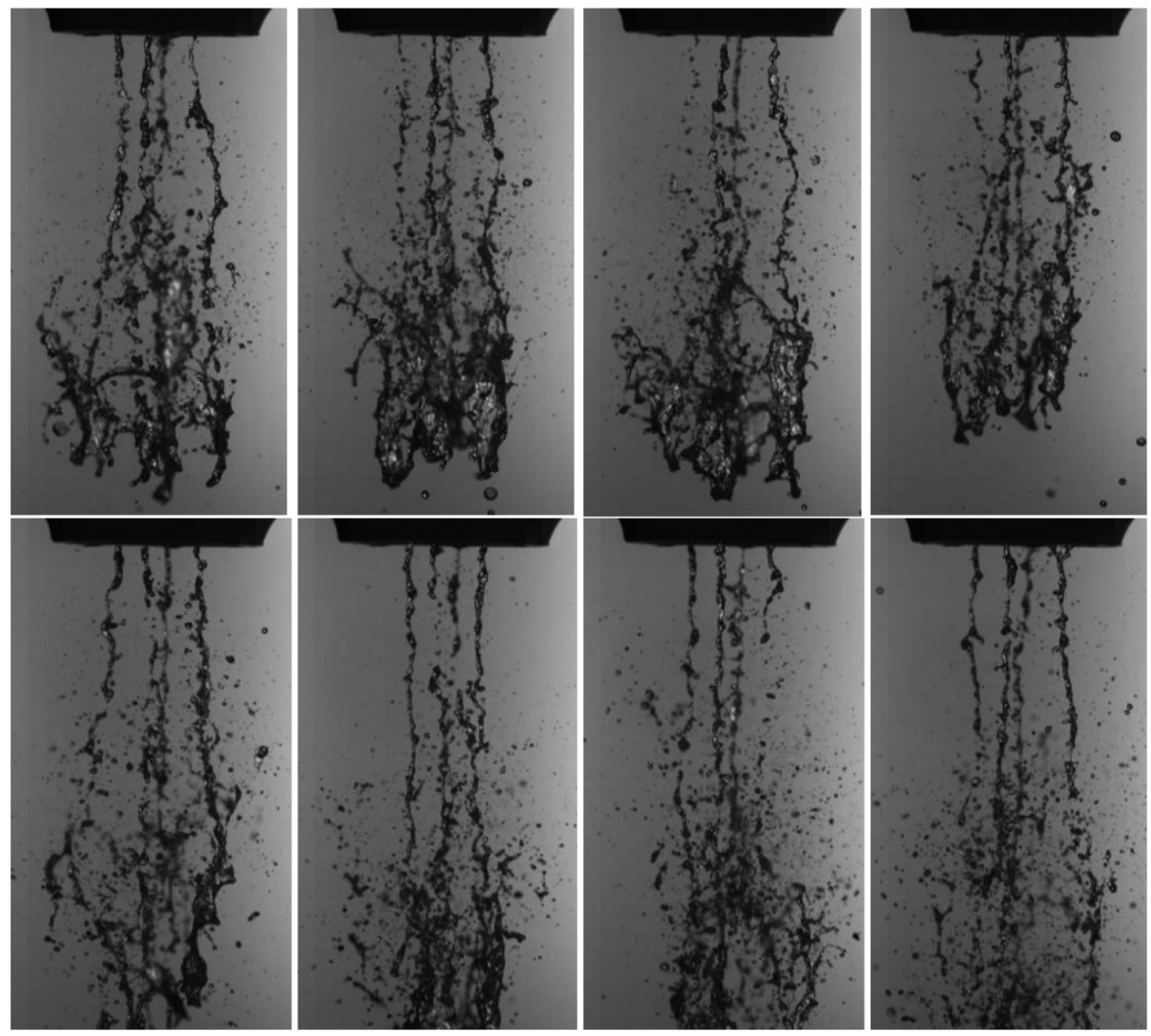

Figure 5. Raw high-speed images of GPI spray acquired continuously at every $12 \mathrm{~ms}$ time interval and at $1.36 \mathrm{~ms}$ after SOI

This visualization work becomes more important and critical, as it assesses the mechanical spring's performance in consistently pumping the fuel at such a high frequency. Continuously captured images corresponding to a single injection event are shown in Fig. 6. The first and third row show the sequential raw images obtained at $40 \mu$ s time step. The second and fourth rows show the corresponding post processed images.

The summary of the the GPI spray evolution is shown in Table 1. The row titled 'Abs. time' refers to the beginning of acquisition as zero and the row titled 'Rel. time' refers to the relative time taking the start of injection (SOI) as zero. After the SOI, the ligaments are formed in the spray and sustain till $3.36 \mathrm{~ms}$. Thereafter ligaments are not seen and only droplets are seen to come out till $7.56 \mathrm{~ms}$. There are no droplets seen after 7.56 and the GPI waits for the next injection event.

Table 1. Spray events at an injection pulse frequency of $83 \mathrm{HZ}$, corresponding to $10,000 \mathrm{rpm}$ of engine.

\begin{tabular}{c|cccc}
\hline & SOI [ms] & End of ligaments [ms] & End of droplets [ms] & Next injection [ms] \\
\hline Abs. time & 9.28 & 12.64 & 16.84 & 21.24 \\
Rel. time & 0 & 3.36 & 7.56 & 12
\end{tabular}




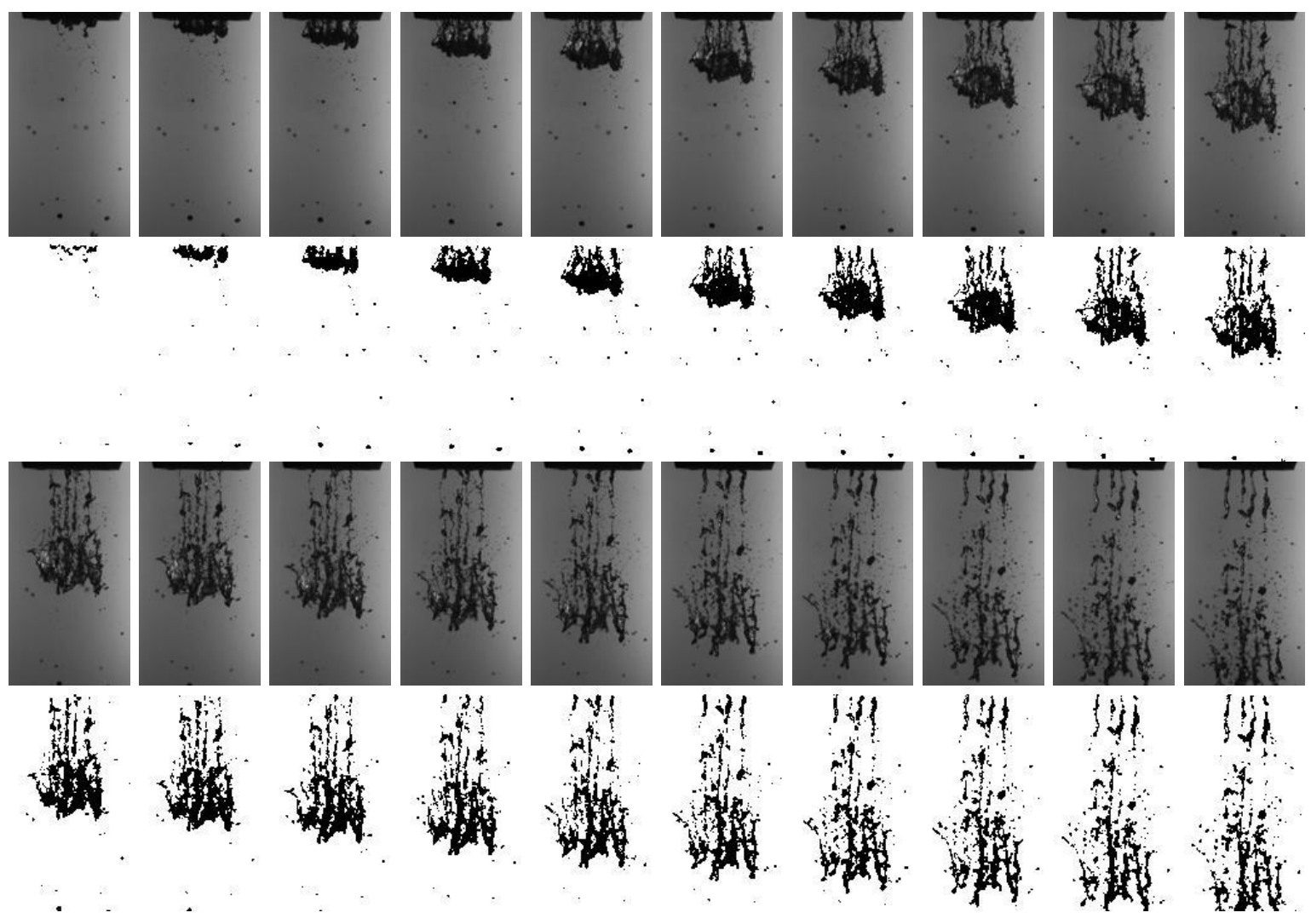

Figure 6. Unprocessed and processed high-speed images of GPI spray acquired at the rate of $25000 \mathrm{fps}$

As the injection is driven by an impulse from the plunger, the spray is seen to eject as a parcel initially, and continues to inject as a jet later, and finally ends with droplets ejection. The later part of the injection event (droplets ejection), is an outcome of the ball valve bouncing back to its home position, due to drop in the fluid pressure above, and assisted by the upwardly-acting spring force below. As the ball valve is closed, the fluid remaining in the injector volume below the ball, exits the nozzle due to gravity, and without pressure assistance from the pump above.

\section{Conclusions}

High-speed back-lit imaging work was successfully carried out on the new-concept gasoline pump injector (GPI). This exercise, demonstrated that this injector can work at a frequency as high as $83 \mathrm{~Hz}$ consistently, and can comply to the demands of its application on a two-wheeler engine (of higher rpms). The spray pattern was found to be very unique as it evolves in two stages, namely ligament stage and droplet stage. The major portion of the injection quantity is seen to get over in less than $4 \mathrm{~ms}$ in the ligament stage and the total injection is seen to get over in less than $8 \mathrm{~ms}$. More than $4 \mathrm{~ms}$ of time is seen to be available, for the preparation of next injection event. The outcome of this visualization work gives enough confidence to apply the GPI on a two-wheeler engine.

\section{Acknowledgements}

This high speed imaging work was carried in the spray research facility of internal combustion engines laboratory at the Indian Institute of Technology Madras (IIT Madras) and we thank Dr.Anand T.N.C. for kindly allowing us to carry out this work there. Manas kumar pal, Keerthi Ganesh and Nithin Tito are acknowledged for their kind help in conducting the experiments at IIT Madras. Our colleagues Sanjay Chadda, Arvind S., Karthick Durairaj, Mark Cavanagh, Rajat Dhara and Mike Plouffe are also acknowledged for their kind support in this project. 


\author{
Abbreviation \\ EFI Electronic fuel injection \\ FPS Frames per second \\ $G P I \quad$ Gasoline pump injector \\ $P F I \quad$ Port fuel injection \\ SOI Start of injection
}

\title{
References
}

[1] Wolfgang Heimberg, Ebersberg; Wolfram Hellmich, Munich; Franz Kogl, Kaufbeuren, all of Germany; Paul Malatinszky, Bulle, Switzerland "Fuel injection device according to the solid-state energy storage principle for internal combustion engines" United states patent US5469828, Date of Patent: Nov. 28, 1995

[2] Mark Cavanagh, Robert Lucas and Yevgeniy Norkin., "Integrated pump and injector for exhaust after treatment," US patent No.US 8,225,602 B2, Date of patent: July 24, 2012.

[3] Mark Cavanagh, Robert Lucas and Yevgeniy Norkin., "Integrated pump and injector for exhaust after treatment," US patent No.US 20100313553A1, Date of patent: Dec 16, 2010.

[4] Balasubramanian Nallannan, Karthick Durairaj, Jayabalan Sethuraman "New-concept Gasoline Pump Injector (GPI) for Motorcycle" SAE Technical Paper 2017-01-0861. 